\title{
Les lipides des poissons d'aquaculture et leurs facteurs de variation
}

\author{
Stéphanie FONTAGNÉ-DICHARRY \\ Françoise MÉDALE
}

\author{
Nutrition, aquaculture et génomique, \\ UMR1067, \\ INRA, \\ 64310 Saint Pée-sur-Nivelle, \\ France \\ $<$ fontagne@st-pee.inra.fr>
}

\begin{abstract}
Fish represent a rich source of the $n-3$ polyunsaturated fatty acids (PUFA), particularly the eicosapentaenoic and docosahexaenoic acids (EPA and DHA) for the human consumer. Although EPA and DHA are more abundant in membrane phospholipids than in triacylglycerols, the content in EPA and DHA generally increases with the lipid content of fish muscle. There are huge differences in muscle lipid content among fish species resulting in great difference in EPA and DHA content. The lipid content of fish flesh varies not only among species, but also within species among individuals depending on age, stage of sexual maturity and also location of fat depots in the muscle. Fish from farming can also be tailored through selective breeding, feeding and rearing practices. Muscle fatty acid profile generally reflects the fatty acid composition of the feed. As EPA and DHA are highly susceptible to attack by oxygen and other organic radicals and resultant damage to PUFA can have serious consequences with potential pathological effects on cells and tissues, an efficient physiological antioxidant protection of farmed fish should also be ensured (e.g., with dietary micronutrients).
\end{abstract}

Key words: aquaculture, fish, flesh, lipids, $n-3$ fatty acids
Le poisson représente une source de protéines et de lipides pour l'alimentation humaine, et sa consommation est recommandée pour ses effets bénéfiques sur la santé. En effet, le poisson constitue la principale source d'acides gras polyinsaturés à longue chaîne (AGPI-LC) n-3 (ou oméga 3) dans I'alimentation humaine. Le poisson provient à la fois de la collecte dans le milieu naturel et de l'élevage. Or, les captures de pêche qui plafonnent depuis une dizaine d'années autour de 90 millions de tonnes ne permettent plus de répondre à l'augmentation de la consommation de poissons au niveau mondial (FAO, 2009). Cette consommation de poissons a plus que doublé depuis 1973 en raison de la croissance de la population mondiale et de l'élévation du niveau de vie dans certains pays asiatiques. Cependant, d'après la $\mathrm{FAO}$, environ $80 \%$ des stocks naturels de poissons sont surexploités ou exploités à leur maximum. Pour répondre à la demande du marché, de plus en plus de produits sont fournis par l'aquaculture. Ce secteur a connu, au cours des dix dernières années, une croissance soutenue de $9 \%$ par an au niveau mondial pour passer de moins d'un million de tonnes au début des années 1950 à 52 millions de tonnes en 2006. Ainsi, la part de poissons consommés issus de l'aquaculture ne cesse d'augmenter: de $6 \%$ en 1970 à $47 \%$ en 2006. Cette proportion élevée est due pour l'essentiel à la Chine où $90 \%$ des poissons consommés proviennent de l'aquaculture, alors que dans le reste du monde cette part représente $24 \%$, mais elle tend à augmenter d'année en année. Le marché de l'aquaculture, dominé par la production asiatique de cyprinidés, offre une grande variété de poissons avec près de 300 espèces différentes. En Europe, la production aquacole est constituée principalement de salmonidés (saumons et truites) et de poissons marins tels que le bar, la daurade et le turbot. Outre cette diversité d'espèces qui se traduit par des différences de sites de dépôt des lipides notamment, la teneur et la qualité des lipides de la chair des poissons d'élevage peuvent varier entre individus, en fonction de la taille, de l'état physiologique du poisson et de son alimentation.

\section{Lipides et sites de dépôt chez les différentes espèces de poissons}

Les lipides des poissons sont riches en AGPI-LC n-3 tels que l'acide eicosapentaénoïque (EPA ; 20:5 n-3) et l'acide docosahexaénoïque (DHA ; 22:6 n-3). Ces acides gras sont retrouvés surtout dans les phospholipides (phosphatidyléthanolamine en particulier), mais les lipides de réserve constitués par les triglycérides sont également relativement riches en AGPI-LC n-3. Alors que la teneur en phospholipides est relativement stable, la teneur en triglycérides est extrêmement variable dans chaque tissu, et l'augmentation des lipides tissulaires est due en très grande partie à l'augmentation de la teneur en triglycérides. Chez les poissons, il existe plusieurs sites de dépôt des lipides tels que le foie, le tissu adipeux périviscéral et le muscle (Sheridan, 1988). L'importance de ces tissus varie suivant les espèces. Le foie constitue le site majeur de stockage des lipides chez les espèces marines comme la morue alors que le tissu adipeux périviscéral est le site de stockage prédominant chez les salmonidés comme la truite. Le muscle, qui représente le principal site de stockage chez l'anguille, peut contenir plus de $20 \%$ de lipides (Henderson et Tocher, 1987). Cette capacité des tissus musculaires à stocker des lipides permet de distinguer différentes catégories de poissons pour le consommateur, avec les espèces maigres, comme la perche du Nil ou la sole dont la teneur en lipides dans le muscle est inférieure à $2 \%$, les espèces intermédiaires qui déposent les lipides dans le muscle (de 2 à $10 \%$ ) et dans $d^{\prime}$ autres sites et les poissons gras, tels que le saumon atlantique, qui ont des teneurs en lipides dans le muscle supérieures à $10 \%$ (tableau 1). Les valeurs rapportées dans le tableau 1 montrent que la quantité d'AGPI-LC $\mathrm{n}-3$ contenue dans $100 \mathrm{~g}$ de chair est proportionnelle à la quantité de lipides du muscle. Plus la chair des poissons est grasse, plus elle apporte d'EPA et de DHA. 
Tableau 1. Exemples de teneurs en lipides et en acides gras de la chair de poissons d'élevage ( $\mathrm{g} / 100 \mathrm{~g}$ de chair) ${ }^{a}$.

\begin{tabular}{|c|c|c|c|c|c|}
\hline Nom commun & Nom scientifique & Lipides & AGPI n-3 & EPA & DHA \\
\hline Perche du Nil & Lates niloticus & 0,3 & 0,14 & 0,02 & 0,08 \\
\hline Sole tropicale & Cynoglossus sp. & 0,3 & 0,13 & 0,04 & 0,06 \\
\hline Tilapia & Oreochromis niloticus & 2 & 0,28 & 0,01 & 0,07 \\
\hline Turbot & Psetta maxima & 4 & 1,07 & 0,36 & 0,43 \\
\hline Bar commun & Dicentrarchus labrax & 4 & 1,26 & 0,44 & 0,58 \\
\hline Dorade royale & Sparus aurata & 5 & 1,24 & 0,33 & 0,56 \\
\hline Truite & Oncorhynchus mykiss & 6 & 1,03 & 0,37 & 0,78 \\
\hline Saumon atlantique & Salmo salar & 13 & 2,28 & 0,61 & 0,87 \\
\hline
\end{tabular}

${ }^{a}$ Ces données sont issues du projet Composition nutritionnelle des produits aquatiques - www.nutraqua.com. Les teneurs représentent les moyennes d'analyses réalisées sur cinq lots différents constitués de dix individus au minimum.

\section{Facteurs de variation de la teneur en lipides musculaires au sein d'une même espèce}

La teneur en lipides des muscles composant les filets, qui représentent les parties consommées chez les poissons, varie non seulement en fonction des espèces mais également, pour une espèce donnée, en fonction des individus. La teneur en lipides augmente avec l'âge et la taille des individus (Henderson et Tocher, 1987), avec par exemple des teneurs en lipides musculaires de 3-4\% chez des saumons de $240 \mathrm{~g}$ et de $17-19 \%$ chez des saumons de 5 kg (Mørkøre et Rørvik, 2001). Au sein même du filet, il existe des variations de teneurs en lipides avec des gradients de dépôts lipidiques dans le muscle pour les espèces intermédiaires et grasses. Ainsi, chez les salmonidés, la partie ventrale du filet est généralement plus grasse que la partie dorsale, et la partie antérieure plus grasse que la partie postérieure (Katikou et al., 2001). Dans le filet, les lipides sont stockés dans des adipocytes dispersés entre les fibres musculaires qui sont particulièrement abondants dans la partie ventrale, au niveau sous-cutané et au sein des cloisons de tissu conjonctif séparant les feuillets musculaires (Zhou et al., 1996).

Les teneurs en lipides musculaires varient également au cours du cycle sexuel. Durant la période de maturation des gonades, les réserves énergétiques stockées dans le muscle sous forme de lipides sont fortement mobilisées et transférées vers les gonades. Cette diminution des teneurs en lipides musculaires (12 versus $5 \%$ chez le saumon atlantique), accompagnée d'une perte de coloration des filets chez les salmonidés, a un impact sur la qualité de la chair (Aksnes et al., 1986). Dans les élevages, ces qualités sont préservées soit en commercialisant les poissons avant la maturation sexuelle (vente au stade portion, souches sélectionnées pour une maturation sexuelle tardive), soit en pratiquant l'élevage avec des animaux stérilisés par l'application d'un choc thermique ou de pression sur les œufs peu après la fécondation, ce qui permet d'obtenir des poissons triploïdes stériles (Taranger et al., 2010).

La teneur en lipides musculaires peut être modulée par l'alimentation chez les espèces grasses et intermédiaires comme la truite. En effet, la teneur en lipides du filet augmente avec la teneur en lipides de l'aliment (figure 1). Les pratiques actuelles en aquaculture tendent à I'utilisation d'aliments haute énergie, riches en lipides. L'apport énergétique supplémentaire sous forme de lipides permet une épargne des protéines alimentaires et une diminution des rejets azotés. Cependant, I'utilisation de tels aliments peut également entraîner un engraissement excessif de la chair des poissons d'élevage. Cette teneur en lipides musculaires peut être diminuée par différentes pratiques d'élevage dans les mois précédant l'abattage, avec soit une période de jeûne ou de restriction alimentaire, soit une période d'alimentation à faible taux de lipides qui n'altère pas le rendement en filet, contrairement au jeûne prolongé (figure 2).

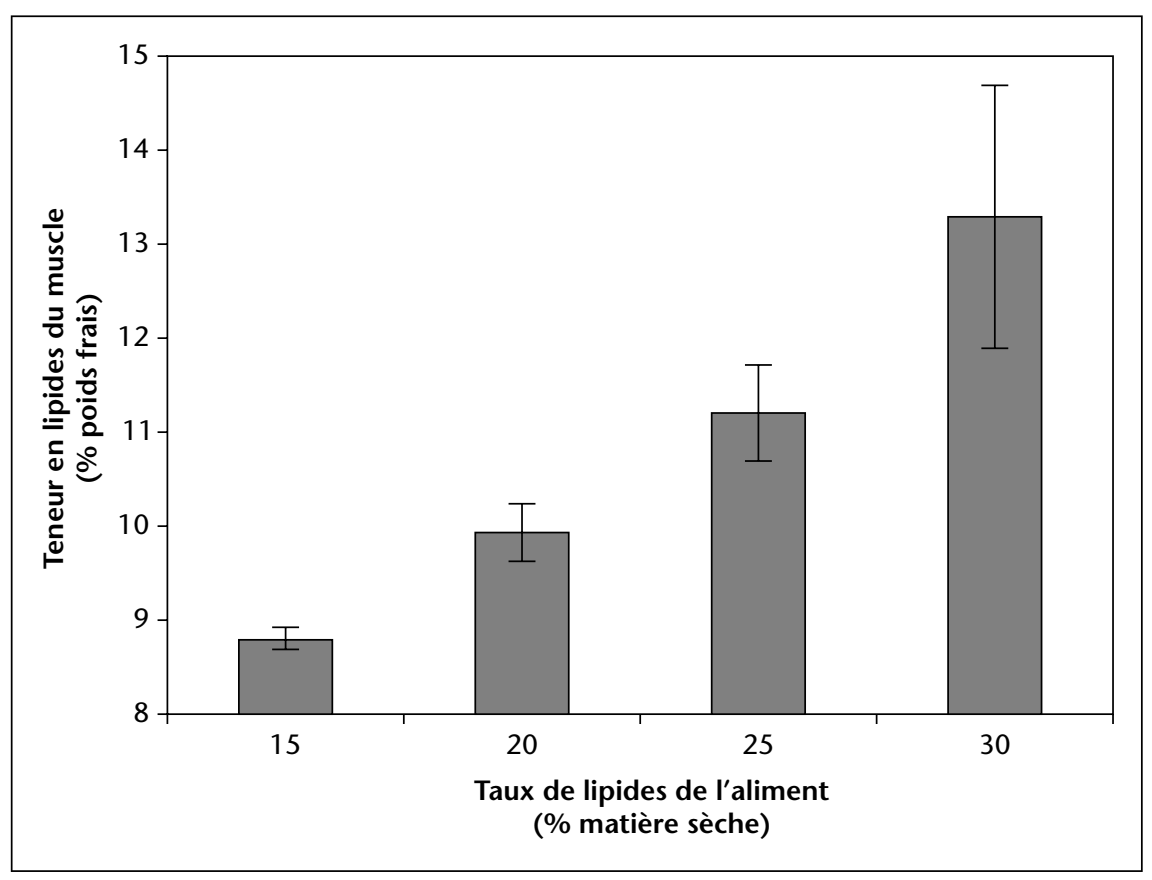

Figure 1. Influence du taux de lipides de l'aliment sur la teneur en lipides du muscle de la truite arc-en-ciel (Gélineau et al., 2001). 
La sélection génétique, qui est une pratique relativement récente en aquaculture, permet également de moduler la teneur en lipides du muscle (Quillet et al., 2005). Chez la truite arcen-ciel, une sélection divergente sur la teneur en lipides du muscle a permis d'obtenir des lignées dont le muscle peut être gras ou maigre avec des variations de l'ordre de 2 à $4 \mathrm{~g}$ pour $100 \mathrm{~g}$ de chair (figure 3). Cependant, la teneur lipidique corporelle globale et la composition en acides gras des lipides du muscle ne sont pas modifiées par la sélection génétique et les proportions d'AGPI-LC n-3 sont semblables

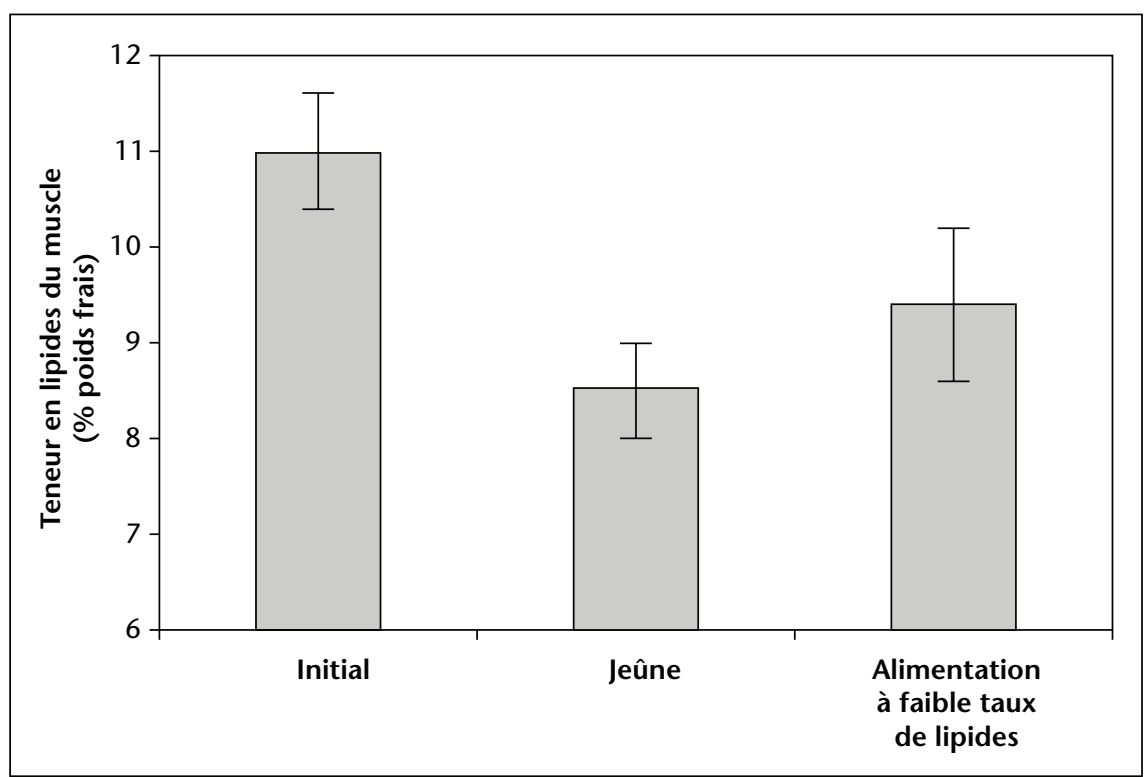

Figure 2. Influence de deux mois de pratiques d'élevage sur la teneur en lipides du muscle de la truite fario préalablement nourrie avec un aliment contenant $26 \%$ de lipides. L'aliment à faible taux de lipides contient $11 \%$ de lipides (Regost et al., 2001).

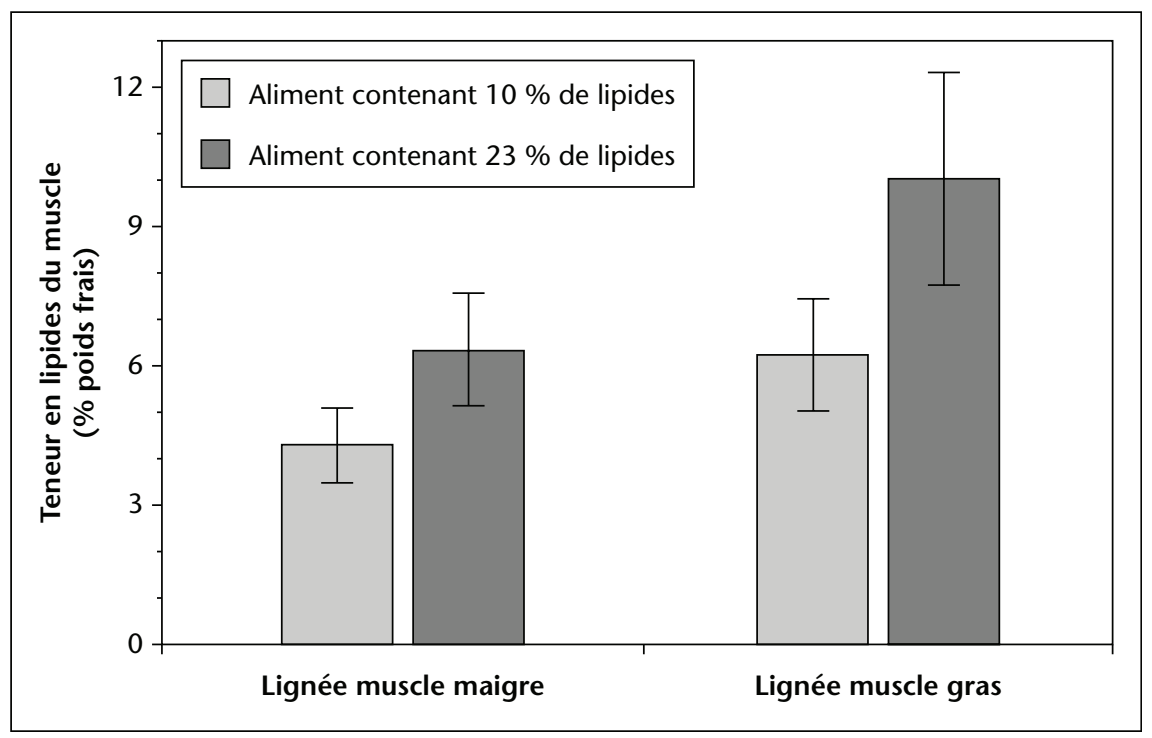

Figure 3. Influence de la sélection génétique et du taux de lipides de l'aliment sur la teneur en lipides musculaires de truites arc-en-ciel issues de la troisième génération de sélection (Kolditz et al., 2008).
Kaushik, 1999). Ainsi, chez les espèces dites intermédiaires ou grasses, les teneurs en EPA et DHA dans le muscle sont d'autant plus importantes que les aliments pour poissons contiennent des quantités croissantes d'AGPILC n-3 apportées par l'huile de poisson. L'essor de l'aquaculture, qui utilise de plus en plus d'aliments composés riches en lipides, a fait augmenter fortement la demande en huile de poisson, principale source de lipides des aliments piscicoles. Actuellement, plus de $90 \%$ de la production mondiale d'huile de poisson est consommée par le secteur de l'aquaculture, dont $55 \%$ uniquement pour les salmonidés (FAO, 2009). En parallèle, la stagnation des captures de pêche limite la disponibilité de I'huile de poisson. L'aquaculture est confrontée aujourd'hui au double défi de réduire l'emploi de matières premières issues de la pêche pour l'alimentation des poissons d'élevage et de conserver à la chair de poisson la valeur nutritionnelle que lui confère sa richesse en EPA et $\mathrm{DHA}$. Les recherches se sont donc intensifiées au cours des dernières années pour évaluer les conséquences du remplacement partiel ou total de l'huile de poisson par des huiles végétales dans les aliments piscicoles. Toutes les études montrent qu'il est possible de substituer, dans l'aliment, de l'huile de poisson par des huiles végétales pendant toute la durée du cycle d'élevage, sans affecter la croissance ou la quantité de lipides déposés dans le muscle, dans la mesure où les besoins en acides gras essentiels sont couverts (Corraze et Kaushik, 2009). Pour les poissons d'eau douce, le besoin en acides gras essentiels peut être couvert par $1 \%$ de 18:3 n-3 ou 0,5\% d'AGPI-LC n-3, alors qu'il est nécessaire d'apporter 0,5 à $1 \%$ d'AGPI-LC $\mathrm{n}-3$ pour les poissons marins qui ont des capacités de bioconversion des acides gras très limitées, voire inexistantes (Corraze et Kaushik, 2009). Cependant, le remplacement de I'huile de poisson par des huiles végétales induit une augmentation des teneurs en acides gras caractéristiques des huiles végétales (de type 18:2 n-6 ou 18:3n-3) et une diminution des teneurs en EPA et DHA dans la chair (tableau 2). Ces modifications de composition en acides gras de la chair engendrées par les régimes à base d'huiles végétales sont néanmoins réversibles avec une alimentation de finition à base $d$ 'huile de poisson. En effet, les teneurs en EPA et DHA peuvent être restaurées et les teneurs en 18:2 n-6 peuvent être diminuées dans la chair des poissons comme cela est rapporté dans le tableau 2 chez le saumon atlantique avec 24 semaines d'alimentation de finition à base d'huile de poisson. Cette stratégie permet de limiter I'utilisation des ressources marines pour l'aquaculture tout en préservant la richesse en AGPI-LC n-3 de la chair des poissons. 
Tableau 2. Composition en acides gras (pourcentage des acides gras totaux) des lipides totaux musculaires de saumon atlantique nourris avec des aliments contenant 25,8 \% d'huile de capelan (FO) ou 25,8\% d'huile de lin (LO) pendant 40 semaines puis nourris avec l'aliment de finition FO pendant 24 semaines. Les lipides totaux représentent $29 \%$ du poids sec des aliments et 8 et $11 \%$ du poids frais des muscles après 40 et 64 semaines d'alimentation (Bell et al., 2004).

\begin{tabular}{|c|c|c|c|c|c|c|}
\hline Acide gras & $\begin{array}{l}\text { Aliment } \\
\text { FO }\end{array}$ & $\begin{array}{l}\text { Aliment } \\
\text { LO }\end{array}$ & $\begin{array}{l}\text { Muscle } \\
\text { FO }\end{array}$ & $\begin{array}{l}\text { Muscle } \\
\text { LO }\end{array}$ & $\begin{array}{l}\text { Muscle } \\
\text { FO-FO }\end{array}$ & $\begin{array}{l}\text { Muscle } \\
\text { LO-FO }\end{array}$ \\
\hline $14: 0$ & 6,3 & 0,4 & 4,7 & 0,7 & 4,8 & 3,6 \\
\hline $16: 0$ & 12,1 & 6,1 & 12,9 & 8,3 & 12,6 & 11,2 \\
\hline 18:0 & 1,1 & 3,1 & 1,9 & 3,6 & 2,1 & 2,6 \\
\hline Somme saturés $^{a}$ & 19,9 & 10,5 & 19,9 & 12,7 & 19,9 & 17,5 \\
\hline $16: 1 \mathrm{n}-7$ & 8,1 & 0,5 & 7,5 & 0,8 & 7,5 & 5,3 \\
\hline 18:1 n-9 & 11,9 & 17,0 & 16,0 & 18,6 & 16,2 & 16,9 \\
\hline 18:1 n-7 & 3,3 & 1,0 & 3,8 & 1,3 & 3,9 & 3,1 \\
\hline 20:1 n-9 & 17,9 & 1,1 & 16,6 & 1,6 & 16,7 & 11,1 \\
\hline $22: 1 n-11$ & 13,3 & 1,1 & 9,9 & 1,1 & 10,3 & 7,2 \\
\hline Somme mono-insaturés ${ }^{b}$ & 58,4 & 21,1 & 57,1 & 24,2 & 57,9 & 46,4 \\
\hline $18: 2 n-6$ & 4,2 & 15,1 & 3,9 & 13,1 & 4,2 & 7,3 \\
\hline $20: 4 n-6$ & 0,2 & 0,1 & 0,3 & 0,1 & 0,2 & 0,2 \\
\hline Somme AGPI n-6 & 5,0 & 15,2 & 4,9 & 14,0 & 5,2 & 8,4 \\
\hline $18: 3 n-3$ & 0,9 & 50,4 & 0,8 & 38,7 & 0,9 & 13,0 \\
\hline $18: 4 n-3$ & 2,9 & 0,2 & 1,5 & 1,2 & 1,4 & 1,4 \\
\hline $20: 3 n-3$ & 0,1 & 0,1 & 0,1 & 2,7 & 0,1 & 1,0 \\
\hline $20: 4 n-3$ & 0,4 & 0,1 & 1,2 & 1,8 & 1,3 & 1,5 \\
\hline $20: 5 n-3$ & 5,9 & 1,0 & 4,3 & 1,3 & 4,1 & 3,4 \\
\hline $22: 5 n-3$ & 0,5 & - & 1,5 & 0,4 & 1,9 & 1,4 \\
\hline $22: 6 n-3$ & 5,0 & 1,5 & 8,1 & 3,1 & 6,6 & 5,5 \\
\hline Somme AGPI n-3 & 15,7 & 53,3 & 17,5 & 49,1 & 16,3 & 27,2 \\
\hline Somme AGPI-LC n-3 & 11,9 & 2,7 & 15,2 & 9,2 & 14,0 & 12,8 \\
\hline
\end{tabular}

a Inclut du 15:0, 17:0, 20:0 et 22:0.

${ }^{b}$ Inclut du 16:1 n-9, 20:1 n-11, 20:1 n-7, 22:1 n-13, 22:1 n-9 et 24:1.

${ }^{c}$ Inclut du 18:3 n-6, 20:2 n-6, 20:3 n-6 et 22:5 n-6.

Des recherches sont également en cours pour évaluer la possibilité de sélectionner des familles performantes pour le stockage d'EPA et DHA comme pour la teneur en lipides musculaires (Médale, 2010).

Afin d'optimiser le dépôt des AGPI-LC n-3 dans la chair du poisson, il est nécessaire de les protéger vis-à-vis de la peroxydation. Du fait du nombre élevé de doubles liaisons, ces acides gras sont particulièrement sensibles à ce phénomène (Hsieh et Kinsella, 1989). La peroxydation des AGPI, qui se produit en présence d'oxygène, de lumière ou de chaleur, conduit à la formation de produits qui peuvent modifier les qualités organoleptiques et sanitaires de la chair
Une des stratégies pour protéger les AGPI-LC $\mathrm{n}-3$ vis-à-vis de la peroxydation lipidique est de supplémenter correctement les aliments avec des molécules antioxydantes afin d'assurer une protection efficace dans I'aliment et in vivo. Ainsi, il a été montré chez la truite arcen-ciel que le besoin en vitamine $E$ pour limiter la peroxydation lipidique musculaire augmentait avec la teneur en AGPI-LC n-3 de l'aliment (Trenzado et al., 2009). D'autres molécules peuvent intervenir et jouer un rôle antioxydant chez les poissons, telles que la vitamine C (Hamre et al., 1997), la vitamine A (Fontagné et al., 2006), les caroténoïdes (Bell et al., 2000) et le sélénium (Lin et Shiau, 2007). Cependant, des problèmes d'équilibre et d'excès peuvent se poser, et des recherches sont nécessaires pour définir les molécules antioxydantes les plus adaptées à l'alimentation des poissons d'élevage.

\section{Conclusion}

Les pratiques d'élevage, en particulier l'alimentation, permettent de moduler la teneur en lipides et la composition en acides gras de la chair des poissons, dans les limites permises par l'espèce. L'addition de substances antioxydantes telles que la vitamine $E$ dans les aliments permet de prévenir la dégradation des AGPI-LC n-3 par peroxydation, contribuant ainsi à conserver la valeur nutritionnelle de la chair de poisson. L'objectif des recherches en cours est de remplacer I'huile de poisson, au sein de I'alimentation de l'élevage, par d'autres matières premières dans un souci de durabilité et d'améliorer le dépôt de l'EPA et du DHA dans la chair du poisson.

\section{RÉFÉRENCES}

Aksnes A, Gjerde B, Roald SO. Biological, chemical and organoleptic changes during maturation of farmed Atlantic salmon, Salmo salar. Aquaculture $1986 ; 53: 7-20$.

Alvarez M], Lopez-Bote C], Diez A, et al. Dietary fish oil and digestible protein modify susceptibility to lipid peroxidation in the muscle of rainbow trout (Oncorhynchus mykiss) and sea bass (Dicentrarchus labrax). Br J Nutr $1998 ; 80$ : 281-9.

Bell JG, McEvoy J, Tocher DR, Sargent JR. Depletion of $\alpha$-tocopherol and astaxanthin in Atlantic salmon (Salmo salar) affects autoxidative defense and fatty acid metabolism. J Nutr $2000 ; 130$ : 1800-8.

Bell JG, Henderson RJ, Tocher DR, Sargent JR. Replacement of dietary fish oil with increasing levels of linseed oil: modification of flesh fatty acid compositions in Atlantic salmon (Salmo salar) using a fish oil finishing diet. Lipids $2004 ; 39$ : 223-32.

Corraze $G$, Kaushik $S$. Les lipides des poisons marins et d'eau douce. OCL $1999 ; 6: 111-5$. 
Corraze G, Kaushik S. Alimentation lipidique et remplacement des huiles de poisson par des huiles végétales en pisciculture. Cah Agric 2009; 18 : 112-8.

FAO. The state of world fisheries and aquaculture 2008 (SOFIA). Rome (Italie): FAO Fisheries and Aquaculture Department, 2009.

Fontagné S, Bazin D, Brèque J, et al. Effects of dietary oxidized lipid and vitamin A on the early development and antioxidant status of Siberian sturgeon (Acipenser baeri) larvae. Aquaculture 2006; 257 : 400-11.

Gélineau A, Corraze G, Boujard T, Larroquet L, Kaushik S. Relation between dietary lipid level and voluntary feed intake, growth, nutrient gain, lipid deposition and hepatic lipogenesis in rainbow trout. Reprod Nutr Dev 2001 ; 41 : 487-503.

Hamre K, Waagbø R, Berge RK, Lie Ø. Vitamins $C$ and E interact in juvenile Atlantic salmon (Salmo salar L.). Free Radic Biol Med 1997 ; 22 : 137-49.

Henderson RJ, Tocher DR. The lipid composition and biochemistry of freshwater fish. Prog Lipid Res 1987 ; $26: 281-347$.
Hsieh RJ, Kinsella JE. Oxidation of polyunsaturated fatty acids: mechanisms, products, and inhibition with emphasis on fish. Adv Food Nutr Res $1989 ; 33$ : 233-341.

Katikou P, Hughes SI, Robb DHF. Lipid distribution within Atlantic salmon (Salmo salar) fillets. Aquaculture $2001 ; 202$ : 89-99.

Kolditz C, Borthaire M, Richard N, et al. Liver and muscle metabolic changes induced by dietary energy content and genetic selection in rainbow trout (Oncorhynchus mykiss). Am J Physiol 2008; 294 : R1154-R1164.

Lin YH, Shiau SY. The effects of dietary selenium on the oxidative stress of grouper, Epinephelus malabaricus, fed high copper. Aquaculture 2007 ; 267 : 38-43.

Médale F. Pratiques d'élevage et qualité nutritionnelle des lipides des poissons. OCL 2010; 17 : 47-51.

Mørkøre T, Rørvik KA. Seasonal variations in growth, feed utilisation and product quality of farmed Atlantic salmon (Salmo salar) transferred to seawater as $0+$ smolts or 1+smolts. Aquaculture 2001; 199: 145-57.
Quillet E, Le Guillou S, Aubin J, Fauconneau B. Two-way selection for muscle lipid content in pan-size rainbow trout (Oncorhynchus mykiss). Aquaculture 2005 ; 245 : 49-61.

Regost C, Arzel J, Cardinal M, Laroche M, Kaushik SJ. Fat deposition and flesh quality in seawater reared, triploid brown trout (Salmo trutta) as affected by dietary fat levels and starvation. Aquaculture $2001 ; 193$ : 325-45.

Sheridan MA. Lipid dynamics in fish: aspects of absorption, transportation, deposition and mobilization. Comp Biochem Physiol 1988 ; 90B : 679-90.

Taranger GL, Carrillo M, Schulz RW, et al. Control of puberty in farmed fish. Gen Comp Endocrinol 2010 ; 165 : 483-515.

Trenzado CE, Morales AE, Palma JM, de la Higuera M. Blood antioxidant defenses and hematological adjustments in crowded/uncrowded rainbow trout (Oncorhynchus mykiss) fed on diets with different levels of antioxidant vitamins and HUFA. Comp Biochem Physiol 2009 ; 149C : 440-7.

Zhou SY, Ackman RG, Morrison C. Adipocytes and lipid distribution in the muscle tissue of Atlantic salmon (Salmo salar). Can J Fish Aquat Sci 1996; 53 : 326-32. 